\title{
Increased BTB-Kelch type substrate adaptor protein immunoreactivity associates with advanced stage and poor differentiation in renal cell carcinoma
}

\author{
HANNA RONKAINEN $^{1}$, MARKKU H. VAARALA ${ }^{1}$, SAILA KAUPPILA ${ }^{2}$, \\ YLERMI SOINI $^{3}$, TIMO K. PAAVONEN ${ }^{4}$, JENNI RASK ${ }^{1}$ and PASI HIRVIKOSKI ${ }^{2}$ \\ ${ }^{1}$ Department of Surgery, Oulu University Hospital, Oulu; ${ }^{2}$ Department of Pathology, \\ Oulu University Hospital, Oulu; ${ }^{3}$ Department of Clinical Pathology, \\ Kuopio University Hospital, Kuopio; ${ }^{4}$ Department of Pathology, \\ Tampere University and Tampere University Hospital, Tampere, Finland
}

Received November 6, 2008; Accepted December 22, 2008

DOI: 10.3892/or_00000383

\begin{abstract}
Renal cell carcinoma (RCC) is a malignancy with increasing incidence. Despite the well-known prognostic factors - the stage, grade and histological subtype - the clinical course of RCC can seem quite random. The aim of this study was to evaluate markers of the oxidative system as candidate prognostic factors for RCC. Our study population consisted of 152 patients who underwent operation for RCC between 1990 and 1999. The tumours were examined with three immunohistochemical markers of the oxidative system, thioredoxin (Trx), NF-E2-related factor (Nfr2) and BTBKelch type substrate adaptor protein (Keap1). Cytoplasmic Keap1 staining was related to poorer prognosis in renal cancer-specific survival. The difference was statistically significant $(\mathrm{P}=0.02)$. Keap1 staining was associated with a more advanced stage and a higher nuclear grade. Cytoplasmic Trx staining was associated with a trend of better prognosis in renal cancer- specific survival. Nfr2 staining was not a prognostic factor in renal cancer-specific survival. In RCC, Keap1 is associated with a more advanced disease, a higher grade and a poorer prognosis.
\end{abstract}

\section{Introduction}

Renal cell carcinoma (RCC) is a malignancy with an increasing incidence and mortality rate (1). At the moment of diagnosis as many as one-third of the patients may have a metastasised disease. After curative treatment about half of the patients will

Correspondence to: Dr Markku H. Vaarala, Department of Surgery, Oulu University Hospital, Oulu, PO Box 21, 90029 OYS, Finland

E-mail:mvaarala@sun3.oulu.fi

Key words: renal cell carcinoma, BTB-Kelch type substrate adaptor protein, thioredoxin, oxidative system, prognosis have a recurrence (2). The stage, Fuhrman grade, histologic subtype and many histological features such as microscopic venous invasion, necrosis and sarcomatoid features are wellknown prognostic factors for RCC $(3,4)$. Many markers associated with angiogenesis, apoptosis and proliferation have been studied as prognostic factors for RCC but no immunohisto-chemical prognostic markers for RCC are in routine use as yet (5). In the era of new treatment possibilities even for metastasised disease there is a definite need for new prognostic factors for RCC so that the treatment and follow-up of the individual patient can be planned better.

Oxidative stress can cause genomic damage and induce carcinogenesis (6). Reactive oxygen species (ROS) are oxygen molecules which have at least one unpaired electron on their outer orbital (7). ROS are involved in a variety of different cellular processes such as apoptosis, cell proliferation and carcinogenesis (6). The potential role of ROS in tumour progression includes the activation of growth factors, hypoxia inducible factor and matrix metalloproteinases and the regulation of apoptosis. There is increased oxidative stress in tumours (8). In many malignancies there is a hypoxic environment which causes alterations in the reduction/ oxidation reaction (redox) state of the cells, increases mitochondrial generation of ROS and induces the stabilisation of the hypoxia inducible factor. Antioxidants and antioxidant enzymes may be important both in normal tissue and cells and malignancies but their importance is not yet well understood (9).

NF-E2-related factor (Nfr2) is a transcription factor which is redox-regulated. $\mathrm{Nfr} 2$ is normally repressed through its cytoplasmic location by binding to the cytoskeleton-associated protein Keap1 (BTB-Kelch type substrate adaptor protein). It has been shown that stresses that activate Nfr2 induce the dissociation of Nfr2 from Keap1. Nfr2 binds to the antioxidant response element (ARE) of genes and controls the expression of an array of detoxification and antioxidant proteins. Keap1$\mathrm{Nfr} 2$ complex is thought to serve as a cytoplasmic sensor of oxidative stress (10). Keap1 is suggested to be a negative regulator of $\mathrm{Nfr} 2$ activity (11). 
Thiol-containing enzyme proteins such as thioredoxins (Trx) are important in maintaining the cellular redox state in cells (12). Thioredoxin is a cysteine containing protein and an antioxidant enzyme which has been shown to be overexpressed in several tumour types and which has prognostic significance (13). It has antioxidative, antiapoptotic and proliferative capacity (14). It has been shown that RCCs which express Trx have a low apoptic index (15).

The aim of this study was to investigate these markers of the oxidative system as candidate prognostic factors for RCC by the use of immunohistochemistry.

\section{Materials and methods}

Patients. Our study population consisted of 152 patients who underwent operation for RCC between 1990 and 1999 at Oulu University Hospital. Seven patients were operated by resection, 145 by radical nephrectomy. The follow-up details were collected from patient records. The follow-up was complete in all cases. The research plan was approved by the local ethics board. The stage of the tumours was assigned by use of the TNM staging of renal cell carcinoma (16).

Tumour samples. Tissue microarrays of formalin-fixed, paraffin-embedded tumours were prepared and used for further analysis. All tumours were reclassified and graded according to the WHO classification by two pathologists (17).

Immunostaining procedure. Paraffin-embedded multi-tissue blocks were cut to $3-\mu \mathrm{m}$ thick sections and mounted on precoated slides. The sections were first deparaffinised in xylene and rehydrated in descending ethanol series. In order to enhance immunoreactivity, the sections were incubated in a citrate buffer ( $\mathrm{pH}$ 6.0) and boiled. Endogenous peroxidise activity was eliminated by incubation in hydrogen peroxide and absolute methanol. The antibodies used in the study were: polyclonal goat IgG antibody to Keap1 (Keap-T E-20 SC-15246 Santa Cruz Biotechology, Inc., dilution 1:100), goat anti-human IgG antibody to Trx (populi No 705 American Diagnostica, Inc., dilution 1:200) and rabbit polyclonal antibody to Nfr2 (Nfr2 C-20: sc-722 Santa Cruz Biotechology, Inc., dilution 1:100). AEC (aminoethylcarbazole) was used as a chromogen.

Immunohistochemical evaluation of the oxidative system markers. Immunohistochemical staining was evaluated simultaneously by four observers (P.H., S.K., Y.S. and H.R.) and a consensus was reached. Immunostaining for cytoplasmic Keap1 was classified as follows: negative, $<10 \%$ positive, $10-40 \%$ positive and $>40 \%$ positive. Immunostaining for nuclear and cytoplasmic Trx was classified negative, $<25 \%$ positive, $25-75 \%$ positive and $>75 \%$ positive. In further analysis for cytoplasmic Keap 1 and Trx, positive subgroups were combined and cytoplasmic immunostaining for Keap1 and Trx was considered positive when staining was detected. Immunostaining for nuclear Nfr2 was classified negative, $<20 \%$ positive, $20-40 \%$ positive and $>40 \%$ positive. Immunostaining for cytoplasmic $\mathrm{Nfr} 2$ was classified negative, $<20 \%$ positive, $20-50 \%$ positive and $>50 \%$ positive.
Table I. Distribution of clinical and tumor stages (TNM classification), histological evaluation and immunostaining for Keap1, Trx and Nfr2.

$\begin{array}{lr}\text { Clinical stage } & \\ \text { I } & 70(46 \%) \\ \text { II } & 12(8 \%) \\ \text { III } & 51(34 \%) \\ \text { IV } & 19(12 \%) \\ \text { Tumour stage } & \\ \text { T1 } & 75(49 \%) \\ \text { T2 } & 12(8 \%) \\ \text { T3 } & 59(39 \%) \\ \text { T4 } & 6(4 \%)\end{array}$

Histological subtype

Clear cell

$134(88.2 \%)$

Papillary

$11(7.2 \%)$

Chromophobic

$5(3.3 \%)$

Unclassified

$2(1.3 \%)$

Fuhrman grading

Grade I

$5(3 \%)$

Grade II

$83(55 \%)$

Grade III

$40(27 \%)$

Grade IV

$22(15 \%)$

Keap1

Negative

$65(45 \%)$

Positive

$79(55 \%)$

$<10 \%$

$10-40 \%$

$44(31 \%)$

$24(17 \%)$

$11(8 \%)$

Cytoplasmic Trx

Negative

$20(14 \%)$

Positive

$123(86 \%)$

$<25 \%$

$25-75 \%$

$14(10 \%)$

$38(27 \%)$

$>75 \%$

$71(50 \%)$

Nuclear Trx

Negative

$32(22 \%)$

Positive

$111(78 \%)$

$<25 \%$

$25-75 \%$

$29(20 \%)$

$46(32 \%)$

$36(25 \%)$

Cytoplasmic Nfr2

Negative

$4(3 \%)$

Positive

$135(97 \%)$

$<20 \%$

$50(36 \%)$

20-50\%

$52(37 \%)$

$>50 \%$

$33(24 \%)$

Nuclear Nfr2

Negative

$26(19 \%)$

Positive

$113(81 \%)$

$<20 \%$

$61(44 \%)$

20-40\%

$38(27 \%)$

$>40 \%$ 


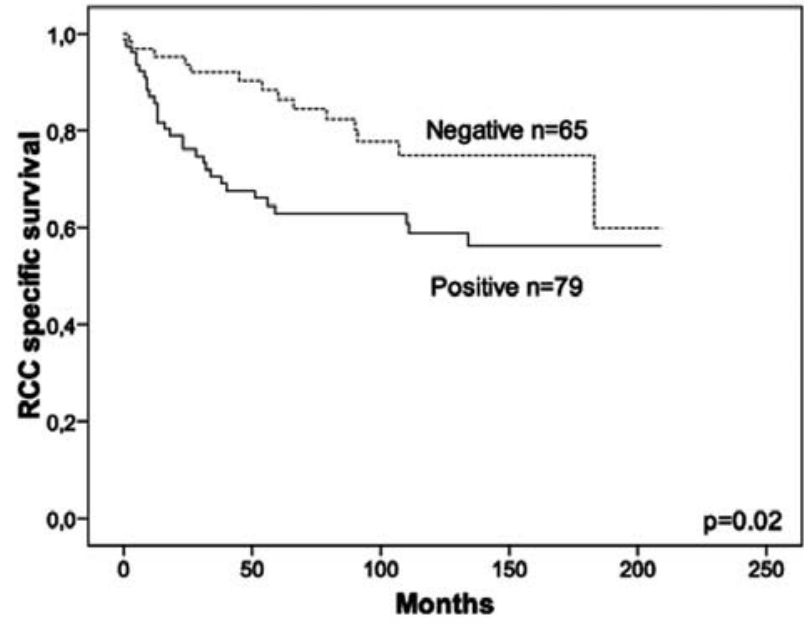

Figure 1. Keap1 as a prognostic factor in RCC-specific survival. Kaplan-Meier curve for survival of 144 patients.

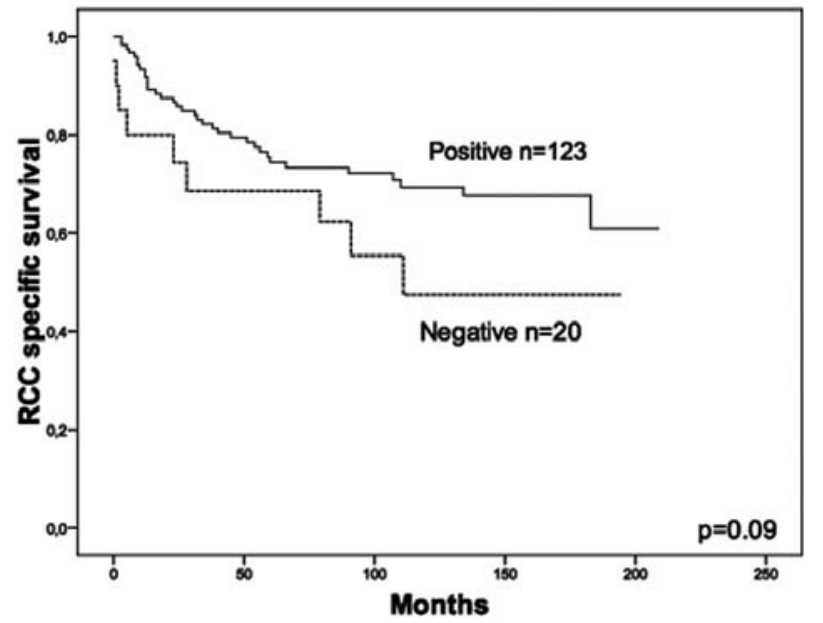

Figure 2. Trx as a prognostic factor in RCC-specific survival. Kaplan-Meier curve for survival of 143 patients.

Statistical analyses. SPSS for Windows 15 (Chicago, IL) was used for statistical analysis. Survival was analysed with the Kaplan-Meier curve and significance of associations with log-rank test. The Cox regression multivariate model was used as in multivariate analysis.

\section{Results}

The median age of the patients was 63 years at the time of diagnosis (range 29-86 years). Seventy-seven (51\%) patients were women, $75(49 \%)$ men. The median follow-up time was 90 months (range 0-209 months). During follow-up, 44 (29\%) patients died of RCC, $40(26 \%)$ died of other causes and $68(45 \%)$ patients were still alive. The distribution of clinical stages and T-classes (TNM classification), the histological evaluation of tumours and the immunostaining for Keap1, Trx and Nfr2 are described in Table I. Sixty-five tumours $(45 \%)$ were negative in cytoplasm immunostaining for Keap1, 79 (55\%) were positive. In cytoplasmic immunostaining for Trx, 20 tumours (14\%) were negative and 123 $(86 \%)$ positive.
Table II. Association between Keap1 immunostaining and stage and grade.

\begin{tabular}{lrr}
\hline & \multicolumn{2}{c}{ Keap1 } \\
\cline { 2 - 3 } & Negative & Positive \\
\hline Stage & & \\
I & $38(58 \%)$ & $27(42 \%)$ \\
II & $7(70 \%)$ & $3(30 \%)$ \\
III & $18(36 \%)$ & $32(64 \%)$ \\
IV & $2(10 \%)$ & $17(90 \%)$ \\
Grade & & \\
I & $3(60 \%)$ & $36(40 \%)$ \\
II & $42(54 \%)$ & $25(66 \%)$ \\
III & $13(34 \%)$ & $15(71 \%)$ \\
IV & $6(29 \%)$ & \\
\hline
\end{tabular}

Table III. Association between Trx immunostaining and stage and grade.

\begin{tabular}{lrr}
\hline & \multicolumn{2}{c}{ Cytoplasmic Trx } \\
\cline { 2 - 3 } & Negative & Positive \\
\hline Stage & & \\
I & $8(12 \%)$ & $57(88 \%)$ \\
II & $2(20 \%)$ & $8(80 \%)$ \\
III & $8(16 \%)$ & $42(84 \%)$ \\
IV & $2(11 \%)$ & $16(89 \%)$ \\
Grade & & \\
I & $0(0 \%)$ & $71(100 \%)$ \\
II & $7(9 \%)$ & $33(87 \%)$ \\
III & $5(13 \%)$ & $14(67 \%)$ \\
IV & $7(33 \%)$ & \\
\hline
\end{tabular}

Keap1 staining was related to poorer prognosis in renal cancer-specific survival (Fig. 1). The difference was statistically significant $(\mathrm{P}=0.02)$. Cytoplasmic Trx staining was associated with a trend of better prognosis in renal cancerspecific survival but the difference was not statistically significant ( $\mathrm{P}=0.09)$ (Fig. 2). Nuclear Trx staining was not a statistically significant prognostic factor in renal cancerspecific survival. Nfr2 staining in neither cytoplasm nor nucleus was a prognostic factor in renal cancer-specific survival. Keap1 staining was associated with a more advanced stage and a higher nuclear grade (Table II). In multivariate analysis with stage and grade, Keap1 staining lost its significance as an independent prognostic factor. Cytoplasmic Trx positivity was associated with lower Fuhrman grade (Table III).

In this material, the stage and grade were both statistically significant prognostic factors in renal cancer-specific survival. In multivariate analysis, the stage seemed to be a statistically significant prognostic factor in renal cancer-specific survival, but Fuhrman grade lost its power as an independent, 
statistically significant prognostic factor. Fuhrman grade was associated with a more advanced stage.

\section{Discussion}

Our study showed that Keap1 staining is related to a poorer prognosis of the RCC patient. Increased Keap1 immunoreactivity was associated with an advanced stage and a higher grade of RCCs, which can largely explain the poorer prognosis of these patients. The Keap1-Nfr2 interaction is thought to be an important regulatory nodal point in the overall response to oxidative or xenobiotic stress. It is assumed that the Keap1Nfr2 complex serves as a cytoplasmic sensor of oxidative stress which is based on the presence of highly reactive sulfhydryl groups in both these proteins (10). It is believed that Keap1 is a negative regulator of Nfr2 activity and either too much or too little Nfr2 activity can result in physiological impairment (11). Cell culture experiments have demonstrated that stresses which activate $\mathrm{Nfr} 2$ induce the dissociation of $\mathrm{Nfr} 2$ from Keap1. When the cell is exposed to inducers of phase II enzymes, the Keap1-Nfr2 complex disrupts and Nfr2 is allowed to translocate into the nucleus. In the nucleus, $\mathrm{Nfr} 2$ binds to the ARE and activates transcription of various detoxification and antioxidant proteins (10). It has recently been demonstrated that loss of Keap1 function activates Nfr2 and provides advantages for lung cancer cell growth (18). In non-small cell lung cancer biallelic inactivation of Keap1 is a frequent genetic alteration which leads to loss of Keap1 function and constitutive activation of $\mathrm{Nfr} 2$-mediated gene expression (19). In breast cancer, a mutation of Keap1 has been found to impair its ability to repress Nfr2 activity (20). Increased expression of Nfr2 has been demonstrated in head and neck squamous cell carcinomas (21).

Despite the association of Keap1 immunoreactivity with a poorer prognosis of the RCC patient, we could not find the same correlation with Nfr2, whose counterpart Keap1 is thought to be. The molecular basis for this observation is unclear. One explanation could be that immunohistochemical quantification of $\mathrm{Nfr} 2$ may not correlate with functionally active Nfr2. Recently it has been demonstrated that cysteine residues have a critical role in maintaining Keap1 function. Modification of cysteine residues by ROS-induced stress allows the dissociation of Nfr2 from Keap1 (22). There are, however, other regulatory mechanisms such as phosphorylation and proteosomal degradation which are also important in regulating Nfr2 activity $(23,24)$. The significance of these mechanisms could not be assessed in this study, although they might explain some of the discrepancy between the detected immunohistochemical staining of Keap1 and Nfr2.

In this study there was a tendency for Trx to be present in tumours of patients with a better prognosis. Trx promotes cell proliferation and it is capable of increasing the growth rate and colony formation of cells (25). Trx can also increase oxidant and drug resistance of various cells (26). A variety of stress stimuli such as hypoxia and photochemical or ultra violet radiation can activate $\operatorname{Trx}(13)$. The expression of Trx has been shown in various tumours. In gastric carcinomas Trx has been shown to associate with increased proliferation and decreased apoptosis (27). On the other hand, in hepatocellular carcinoma, high Trx expression is related to decreased cell proliferation (28). In small-cell lung carcinoma, Trx is highly expressed and takes part in activation of transcriptional factors and regulates apoptosis. In high grade tumours, Trx expression is diminished, suggesting loss of redox regulation (29). Trx expression has also been described in astrosytic brain tumours where it may influence the biological behaviour of the tumour, possibly modulating cell proliferation and necrosis of the tumour (30). In earlier studies it has been shown that Trx positivity associates with lower apoptotic index in RCC (15). It would be reasonable to suppose that these patients would have a better prognosis. In this study, Trx was related to a better prognosis of the RCC patient. The difference was not, however, statistically significant. We suppose that our study population is too small to show a statistically significant difference in survival with Trx staining but it is still possible that the correlation exists.

The advantages of our study were a long follow-up time and detailed follow-up information. Our study population was large enough to prove the effect of the traditional prognostic factors - the stage and Fuhrman grade. Histological subtype, however, seemed not to be a statistically significant prognostic factor, presumably because of the small number of patients with histology other than clear cell carcinoma.

The oxidative system is involved in RCC and it may be that in the future there will be markers of oxidative stress or the antioxidative system which can help us assess the prognosis of RCC patients. In RCC, Keap1 is related to a more advanced disease, a higher grade and a poorer prognosis.

\section{Acknowledgements}

We would like to thank Manu Tuovinen and Riitta Vuento for their skillfull technical assistance, Pasi Ohtonen, M.Sc., for assistance with statistical analyses and Oulu University, Oulu University Hospital and Finnish Urological Association for financial support.

\section{References}

1. Pantuck AJ, Zisman A and Belldegrun AS: The changing natural history of renal cell carcinoma. J Urol 166: 1611-1623, 2001.

2. Bui MH, Zisman A, Pantuck AJ, et al: Prognostic factors and molecular markers for renal cell carcinoma. Expert Rev Anticancer Ther 1: 565-575, 2001.

3. Cheville JC, Lohse CM, Zincke H, et al: Comparisons of outcome and prognostic features among histologic subtypes of renal cell carcinoma. Am J Surg Pathol 27: 612-624, 2003.

4. Lang H, Lindner V, Letourneux H, et al: Prognostic value of microscopic venous invasion in renal cell carcinoma: Long-term follow-up. Eur Urol 46: 331-335, 2004.

5. George $S$ and Bukowski RM: Biomarkers in clear cell renal cell carcinoma. Expert Rev Anticancer Ther 7: 1737-1747, 2007.

6. Mates JM, Segura JA, Alonso FJ, et al: Intracellular redox status and oxidative stress: Implications for cell proliferation, apoptosis, and carcinogenesis. Arch Toxicol 82: 273-299, 2008.

7. Fridovich I: Oxygen toxicity: A radical explanation. J Exp Biol 201: 1203-1209, 1998

8. Oberley TD: Oxidative damage and cancer. Am J Pathol 160: 403-408, 2002.

9. Zelko IN, Mariani TJ and Folz RJ: Superoxide dismutase multigene family: A comparison of the CuZn-SOD (SOD1), mn-SOD (SOD2), and EC-SOD (SOD3) gene structures, evolution, and expression. Free Radic Biol Med 33: 337-349, 2002.

10. Itoh K, Wakabayashi N, Katoh Y, et al: Keap1 represses nuclear activation of antioxidant responsive elements by Nrf2 through binding to the amino-terminal Neh2 domain. Genes Dev 13: 76-86, 1999. 
11. Liu H, Colavitti R, Rovira II, et al: Redox-dependent transcriptional regulation. Circ Res 97: 967-974, 2005.

12. Arner ES and Holmgren A: Physiological functions of thioredoxin and thioredoxin reductase. Eur J Biochem 267: 6102-6109, 2000.

13. Powis G and Montfort WR: Properties and biological activities of thioredoxins. Annu Rev Pharmacol Toxicol 41: 261-295, 2001.

14. Powis G, Mustacich D and Coon A: The role of the redox protein thioredoxin in cell growth and cancer. Free Radic Biol Med 29: 312-322, 2000.

15. Soini Y, Kallio JP, Hirvikoski P, et al: Antioxidant enzymes in renal cell carcinoma. Histol Histopathol 21: 157-165, 2006.

16. UICC (2002): TNM Classification of Malignant Tumours. 6th edition. Wiley \& Sons, New York, 2002.

17. IARC (2004): Tumours of the Urinary System and Male Genital Organs. IARC Press, Lyon, 2004

18. Ohta T, Iijima K, Miyamoto M, et al: Loss of Keap1 function activates Nrf2 and provides advantages for lung cancer cell growth. Cancer Res 68: 1303-1309, 2008.

19. Singh A, Misra V, Thimmulappa RK, et al: Dysfunctional KEAP1-NRF2 interaction in non-small-cell lung cancer. PLoS Med 3: e420, 2006.

20. Nioi P and Nguyen T: A mutation of Keap1 found in breast cancer impairs its ability to repress Nrf2 activity. Biochem Biophys Res Commun 362: 816-821, 2007.

21. Stacy DR, Ely K, Massion PP, et al: Increased expression of nuclear factor E2 p45-related factor 2 (NRF2) in head and neck squamous cell carcinomas. Head Neck 28: 813-818, 2006.

22. Yamamoto T, Suzuki T, Kobayashi A, et al: Physiological significance of reactive cysteine residues of Keap1 in determining Nrf2 activity. Mol Cell Biol 28: 2758-2770, 2008.
23. McMahon M, Itoh K, Yamamoto M, et al: Keap1-dependent proteasomal degradation of transcription factor Nrf2 contributes to the negative regulation of antioxidant response element-driven gene expression. J Biol Chem 278: 21592-21600, 2003.

24. Apopa PL, He X and Ma Q: Phosphorylation of Nrf2 in the transcription activation domain by casein kinase 2 (CK2) is critical for the nuclear translocation and transcription activation function of Nrf2 in IMR-32 neuroblastoma cells. J Biochem Mol Toxicol 22: 63-76, 2008

25. Powis G, Kirkpatrick DL, Angulo M, et al: Thioredoxin redox control of cell growth and death and the effects of inhibitors. Chem Biol Interact 111-112: 23-34, 1998.

26. Sasada T, Nakamura H, Ueda S, et al: Possible involvement of thioredoxin reductase as well as thioredoxin in cellular sensitivity to cis-diamminedichloroplatinum (II). Free Radic Biol Med 27: 504-514, 1999.

27. Grogan TM, Fenoglio-Prieser C, Zeheb R, et al: Thioredoxin, a putative oncogene product, is overexpressed in gastric carcinoma and associated with increased proliferation and increased cell survival. Hum Pathol 31: 475-481, 2000.

28. Rubartelli A, Bonifaci N and Sitia R: High rates of thioredoxin secretion correlate with growth arrest in hepatoma cells. Cancer Res 55: 675-680, 1995.

29. Soini Y, Kahlos K, Napankangas U, et al: Widespread expression of thioredoxin and thioredoxin reductase in non-small cell lung carcinoma. Clin Cancer Res 7: 1750-1757, 2001.

30. Haapasalo H, Kylaniemi M, Paunul N, et al: Expression of antioxidant enzymes in astrocytic brain tumors. Brain Pathol 13: $155-164,2003$ 